\title{
Editorial
}

\section{Supersymmetry, Supergravity, and Superstring Phenomenology}

\author{
Shaaban Khalil, ${ }^{1}$ Gordon Kane, ${ }^{2}$ Ignatios Antoniadis, ${ }^{3,4}$ and Stefano Moretti ${ }^{5}$ \\ ${ }^{1}$ Center for Fundamental Physics, Zewail City of Science and Technology, Giza, Egypt \\ ${ }^{2}$ Department of Physics, University of Michigan, Ann Arbor, MI, USA \\ ${ }^{3}$ Department of Physics, LPTHE, Sorbonne Université, UPMC, Paris, France \\ ${ }^{4}$ University of Bern, Bern, Switzerland \\ ${ }^{5}$ Department of Physics, University of Southampton, Southampton, UK \\ Correspondence should be addressed to Shaaban Khalil; skhalil@zewailcity.edu.eg
}

Received 14 December 2016; Accepted 14 December 2016

Copyright (C) 2016 Shaaban Khalil et al. This is an open access article distributed under the Creative Commons Attribution License, which permits unrestricted use, distribution, and reproduction in any medium, provided the original work is properly cited. The publication of this article was funded by $\mathrm{SCOAP}^{3}$.

Supersymmetry, supergravity, and superstring are amongst the most popular research topics in particle physics. Supersymmetry is a generalization of the space-time symmetries of quantum field theory that links the matter particles with the force-carrying particles and implies that there are additional superparticles necessary to complete the symmetry. Supergravity is the theory that combines the principles of supersymmetry and general relativity. It naturally includes gravity along with the other fundamental forces (the electromagnetic force, the weak nuclear force, in turn already unified in the electroweak interactions, and the strong nuclear force). String theory is the leading candidate for a theory that unifies all fundamental forces in nature in a consistent scheme. It also provides a consistent framework for the theory of quantum gravity. Compactified string/M-theories make testable predictions about our four-dimensional world.

The phenomenology of supersymmetry, supergravity, and superstring is thus very rich and covers many topics: flavour physics and CP violation, Higgs and collider physics, model building beyond the Standard Model, and astroparticle physics and cosmology. Some recent developments in these theories, each with important applications to particle physics and/or cosmology, are the main theme of this special issue.

One of the papers of this special issue discusses the constrained generalized Killing spinors, which characterize supersymmetric flux compactifications of supergravity theories, using geometric algebra techniques. Another paper presents a study on what are called Adinkras, which are combinatorial objects developed to study (1-dimensional) supersymmetry representations. Another paper reviews moduli stabilization in type IIB string theory compactification with fluxes. Another paper in this special issue describes supermultiplets wherein a continuously variable "tuning parameter" modifies the supersymmetry transformations. Another paper studies the constraints imposed on the Minimal Supersymmetric Standard Model (MSSM) parameter space by the Large Hadron Collider (LHC) Higgs mass measurements and gluino mass lower bound. Another paper studies helical phase inflation which realizes "monodromy inflation" in supergravity theory. Another paper considers scalar Wilson operators of $N=4$ Supersymmetric Yang-Mills (SYM) theories at high spin and generic twist operators in the multicolor limit. Another paper author gives an overview about the features that the Mathematica package SARAH provides to study new supersymmetric models. Another paper reviews a possible mechanism for the spontaneous breaking of supersymmetry, based on the presence of vacuum condensates. Another paper constructs and studies a formulation of a chargeless complex vector matter field in a supersymmetric framework.

Shaaban Khalil Gordon Kane Ignatios Antoniadis Stefano Moretti 

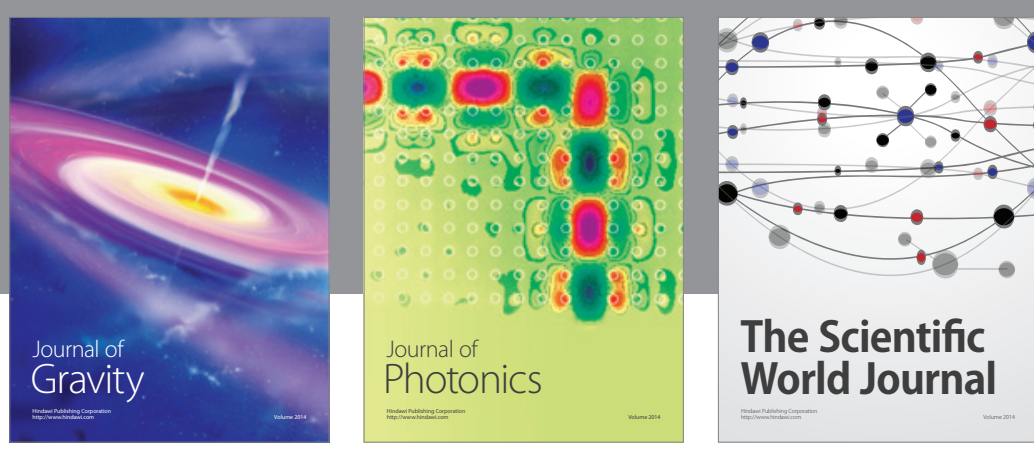

The Scientific World Journal
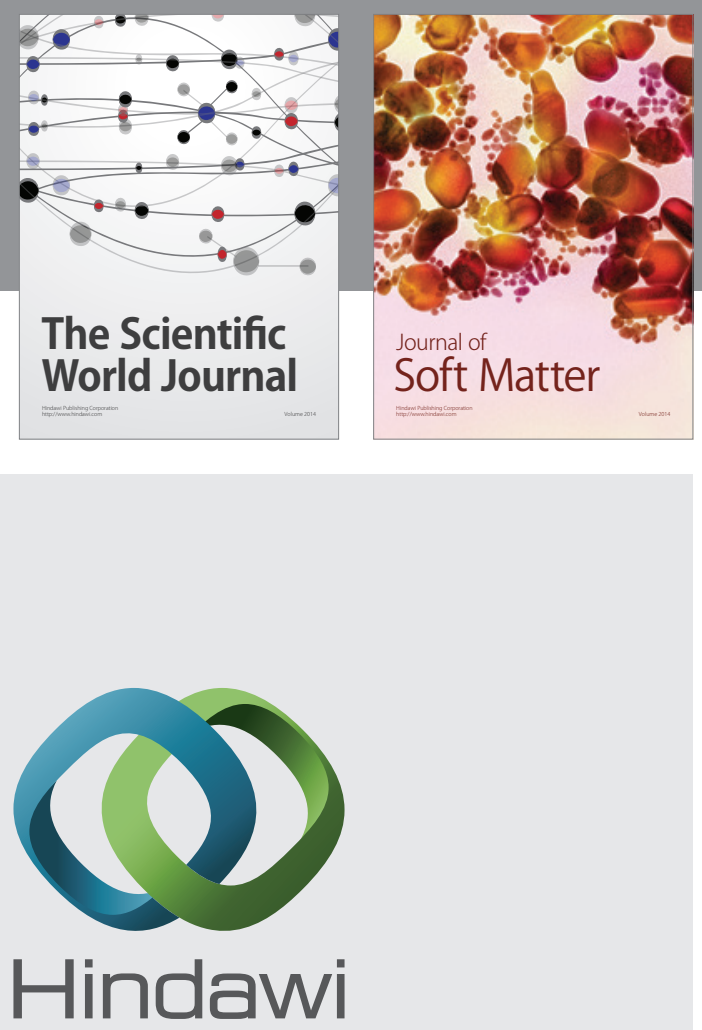

Submit your manuscripts at

http://www.hindawi.com

nternational Journal of

Statistical Mechanics
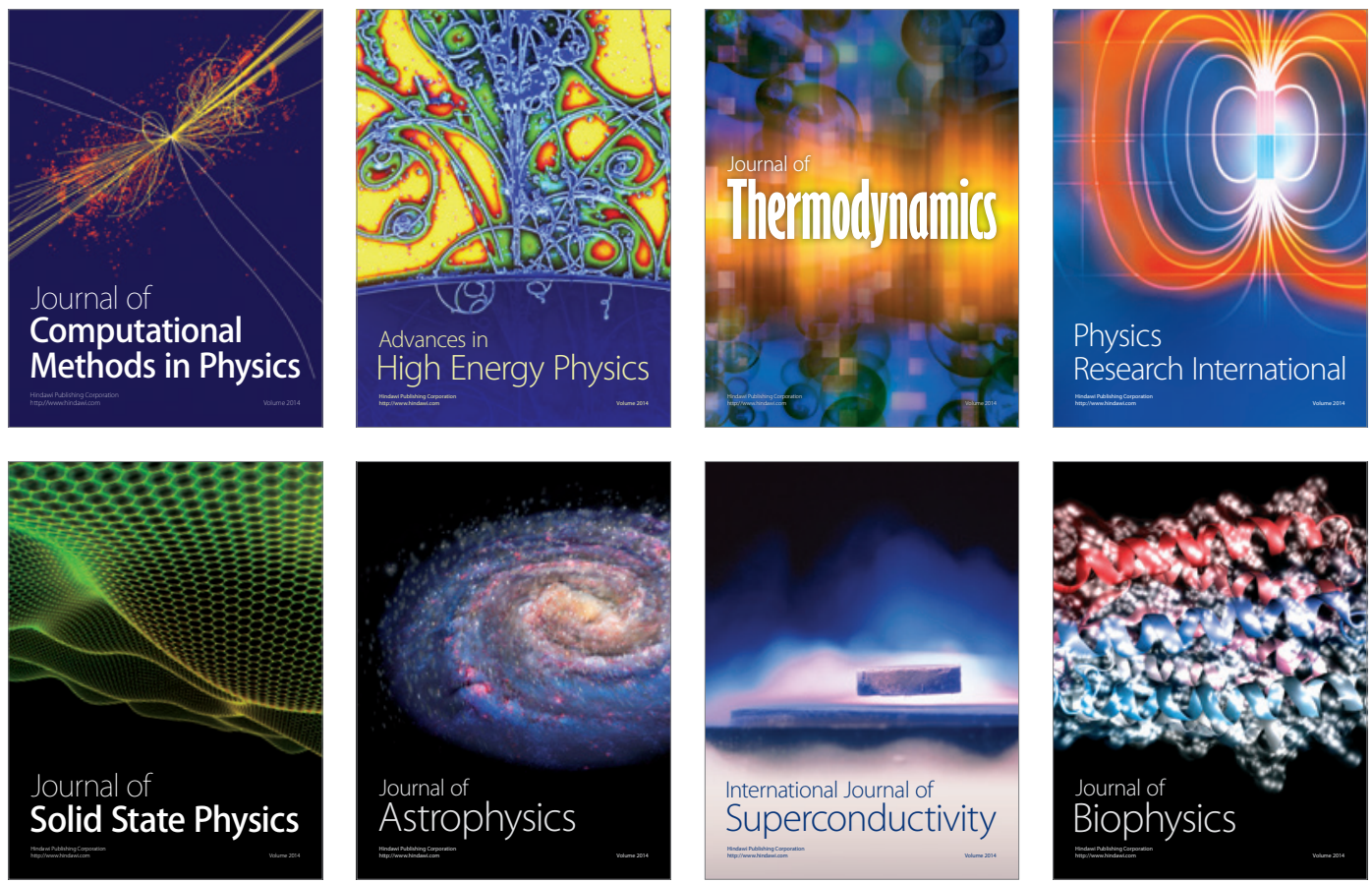
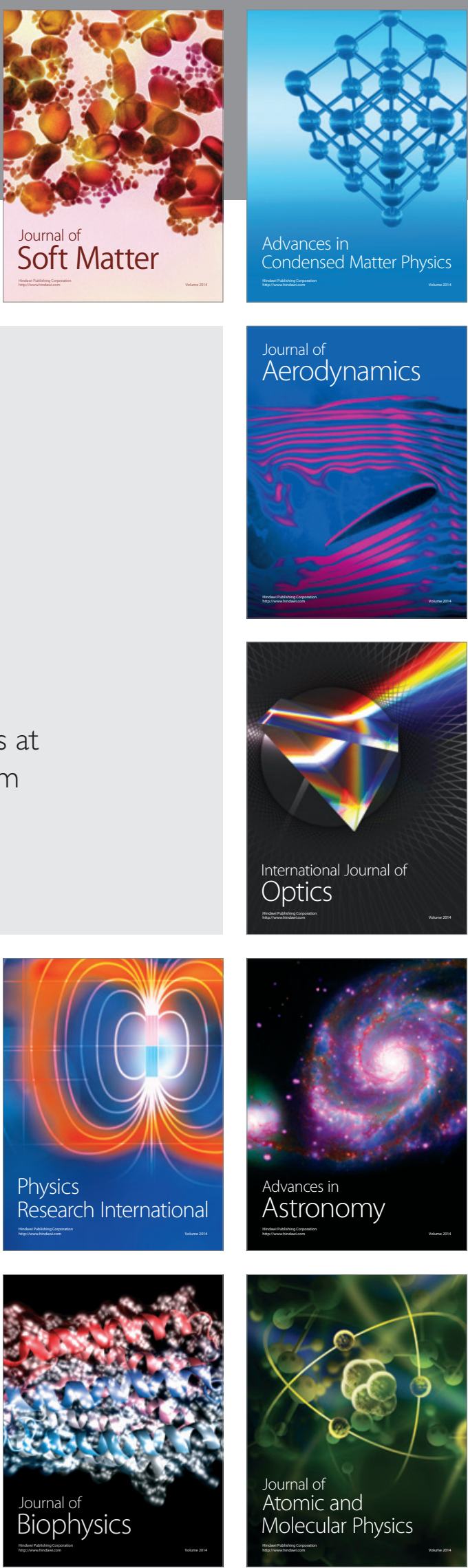